\title{
Ambiente de Acompanhamento e Controle de Avaliação de Aprendizado em Plataforma de Ensino Virtual
}

\author{
Wellington Pacheco ${ }^{1}$, Martony Demes ${ }^{1}$, James da L. Dias ${ }^{1}$ \\ Tardelly Cavalcante $^{1}$, André Soares ${ }^{1}$, Jordão Frazão ${ }^{1}$, Anselmo Paiva ${ }^{2}$ \\ ${ }^{1}$ Universidade Federal do Piauí (UFPI) - Teresina - PI - Brazil \\ ${ }^{2}$ Universidade Federal do Maranhão (UFMA) - São Luís - MA - Brazil \\ pacheco@ufpi.edu.br, \{mardemes, jamesdias0, tardellycavalcante\}@gmail.com \\ jordaofrazao@gmail.com, andre.soares@ufpi.edu.br, paiva@nca.ufma.br
}

\begin{abstract}
Virtual learning environments provide an alternative to distance learning. This was notable in this period of pandemic and social isolation. The challenge, however, is in the application of student assessment, which is due to the need to supervise the application of online assessments and to avoid fraud attempts. Therefore, this article presents a proposal for digital monitoring of the exam application. The results suggest a possibility to control and supervise the application of exams in the context of remote learning.
\end{abstract}

Resumo. Os ambientes virtuais de ensino proporcionam uma alternativa para a modalidade a distância. Isso notabilizou-se nesse período de pandemia e isolamento social. O desafio, entretanto, está na aplicação da avaliação dos estudantes, o que se deve à necessidade de supervisionar a aplicação de avaliações online e evitar tentativas de fraudes. Para tanto, esse artigo apresenta uma proposta de monitoramento digital de aplicação de exame online. Os resultados sugerem uma possibilidade de controlar e fiscalizar a aplicação de exames no contexto do ensino remoto.

\section{Introdução}

Os ambientes de ensino virtuais são uma alternativa de aprendizagem para as modalidades a distância. Existem outras denominações: educação online, híbrida ou remota. Essas formas de ensino oportunizaram cursos e formações para diversos locais remotos em todo o mundo. Isso é possível por meio de conexão com a internet e uso de plataformas ou navegadores que disponibilizem o conteúdo que será consumido pelo aluno a partir do computador ou dispositivo móvel (tablet, smartphone etc).

Nesse cenário, a educação online revolucionou o ensino especialmente com a inserção de plataformas específicas para modalidades: Cursos Online Abertos e Massivos (MOOCs), Coursera, EdX, Udacity, Moodle, entre outras [Indi et al. 2021]. Essas ferramentas ou portais de ensino possibilitam o acesso a cursos de qualidade e de renomadas instituições internacionais (MIT, Stanford University, centenas de Universidades pelo Brasil, entre outras).

Outro aspecto relevante é que esse cenário de educação online acentuou-se nesse período devido à necessidade de isolamento social em virtude da Covid-19 
[Vieira and Silva 2020]. Assim, o número de cursos online e de estudantes nessas plataformas cresceu exponencialmente [Indi et al. 2021].

Diante desse contexto, cursos e graduações, no âmbito da modalidade online, também exigem avaliações e certificações para que os discentes concluam suas formações. Para tanto, faz-se necessário o uso de mecanismos para supervisionar a aplicação das provas online. E isso ainda é um desafio a ser superado dado que não há uma fiscalização presencial e fato este que pode gerar tentativas de fraudes, necessidade de autenticar efetivamente o aluno e controlar tentativas de trapaças [Prathish et al. 2016]. Além disso, todo esse monitoramento deve ser automatizado dada a quantidade significativa de alunos em uma determinada turma.

Nessa perspectiva, o objetivo deste trabalho é apresentar uma estratégia que auxilie o professor a gerenciar a aplicação dos exames online no contexto do ensino remoto. Para isso, é proposto um ambiente digital para controle do comportamento do estudante durante a realização do exame. Tal controle é composto por: i) monitoramento da janela do navegador que consta o exame aplicado e ii) monitoramento do olhar durante a realização do exame. Nessa perspectiva, será utilizado a webcam durante os exames para capturar imagens que serão classificadas em Redes Neurais Convolucionais (CNN) e confrontadas com as informações extraídas do monitoramento do navegador. O diferencial desta pesquisa é a possibilidade de uso de exame online em qualquer navegador. Isso permite uso de diversas ferramentas gratuitas online e utilizadas significativamente como o Google Forms. Além disso, esse estudo realizou testes com o ambiente digital proposto com estudantes de curso superior em uma prova real e online.

\section{Trabalhos Relacionados}

Métodos de monitoramento durante aplicação de exames online podem ser realizados com base no movimento dos olhos e por meio do monitoramento da tela ativa do computador ou dispositivo móvel. Dentro do conjunto dessas informações pode-se inferir indícios de comportamentos indesejados dentro do contexto de uma prova online. Nesse prisma, há pesquisas que exploraram alguns desses elementos de controle.

A pesquisa de [Muzaffar et al. 2021] apresenta um levantamento bibliográfico de trabalhos relacionados ao tema ilustrando principais pesquisas sobre técnicas utilizadas em supervisão de exames online. O estudo ilustra os algoritmos de redes neurais mais utilizados, as ferramentas, os datasets e as soluções apresentadas entre 2016 e 2020.

Nessa perspectiva, algumas pesquisas serão expostas neste artigo com propósito de cotejar características evidenciando o diferencial explorado nesta pesquisa. Investigouse também que trabalhos fazem uso de ambiente web e com possibilidade de aplicar a ferramenta Google Forms.

O estudo de [Indi et al. 2021] apresenta um modelo de um sistema de foco visual de atenção do aluno que faz detecção de movimento ocular considerado inadequado em contexto de exame online. Porém, a pesquisa não aborda monitoramento da janela ativa e não faz uso de navegador web. O referido estudo não fez um experimento com estudantes. Não obstante, a acurácia (grau de exatidão) da rede neural utilizada é acima de $90 \%$.

Por outro lado, a pesquisa de [Prathish et al. 2016] apresenta uma proposta que faz captura de movimento ocular e o monitoramento da tela ativa com registro de mudança 
desta tela. O diferencial é captura de áudio e de objetos. Além disso, a acurácia é de $80 \%$.

Outra investigação relevante é o estudo de [Asep and Bandung 2019]. Nessa pesquisa, é proposto um sistema próprio que também faz detecção de movimento ocular. Porém, não faz detecção de janela ativa na tela. O diferencial é ser proposto para ambiente em dispositivos móveis (android).

Já o estudo [Hu et al. 2018] propõe um sistema que obtém informações da postura da cabeça e da boca bem como a verificação do comportamento anormal dos alunos durante o exame online. Também não fez o monitoramento de captura da tela.

Recentemente, a pesquisa de [Li et al. 2021] apresenta uma abordagem para supervisionar provas online por meio de captura de rosto, olhos, tela e mouse. Também é investigado o uso de monitoramento de tela ativa. O diferencial deste trabalho é o uso de ambiente por meio de navegador web com a instalação de $\operatorname{plugin}(*)$ e teve uma aplicação com 24 estudantes.

Diante desse cenário, sumarizou-se as principais características exploradas nos trabalhos citados no contexto de supervisão de provas online na Tabela 1 a seguir. Para simplificar a exibição da tabela, considere: DMOI - Detecção de Movimento Ocular Inapropriado; MJA - Monitoramento de Janela Ativa; UNW - Uso de Navegador Web; UGF - Uso do Google Forms; AE - Aplicação em Estudantes

Tabela 1. Artigos sobre supervisão de exames online

\begin{tabular}{|l|c|c|c|c|c|}
\hline Artigos & DMOI & MJA & UNW & UGF & AE \\
\hline$[$ Indi et al. 2021 $]$ & Sim & Não & Não & Não & Não \\
\hline$[$ Prathish et al. 2016 $]$ & Sim & Sim & Não & Não & Não \\
\hline$[$ Asep and Bandung 2019] $]$ & Sim & Não & Não & Não & Não \\
\hline$[$ Hu et al. 2018 $]$ & Sim & Não & Não & Não & Não \\
\hline$[$ Li et al. 2021 $]$ & Sim & Sim & Sim* & Não & Sim \\
\hline Proposta desta pesquisa & Sim & Sim & Sim & Sim & Sim \\
\hline
\end{tabular}

Diante do exposto, verifica-se que todos os trabalhos fazem detecção de movimento ocular. Não obstante, apenas duas pesquisas investigaram o monitoramento de janela (ou tela) ativa. Vale destacar que apenas uma pesquisa avaliou estudantes e em nenhuma utilizou-se a ferramenta Google Forms. Tal ferramenta é gratuita e utilizada significativamente.

\section{Proposta}

Os trabalhos discutidos na seção anterior evidenciam dois aspectos importantes: a detecção do movimento ocular do estudante e o monitoramento da tela vigente da prova. Com base nisso, o modelo proposto neste trabalho é dividido em duas partes: i) captura de imagens controladas e rotuladas para o treinamento do modelo da rede neural e ii) a captura de tela da janela ativa do navegador com o exame online. Na Seção 4 serão apresentados mais detalhes desse modelo.

Além disso, foi realizado um experimento com 13 estudantes conectados à internet por meio de notebooks com webcams ligadas. Durante a aplicação da prova foram capturadas quatro imagens por segundo. Logo em seguida foi aplicada uma classificação 
com redes neurais com as imagens capturadas. Esse procedimento objetivou identificar se o usuário estava olhando para a tela do computador bem como se estava na janela do exame a ser realizado. Nas seções a seguir serão descritos todos os procedimentos realizados.

\subsection{Ambiente Digital}

O grupo desta pesquisa desenvolveu um ambiente digital que possibilita a inserção de aula virtual e gerenciamento de alunos e tarefas (provas online). Por meio desse ambiente será possível monitorar os movimentos faciais, oculares e a tela (janela do navegador). Nesse quadro, os exames online podem ser inseridos por meio de links de formulários do Google Forms. O ambiente digital foi denominado Sentinela e está acessível por meio do link Sentinela. A Figura 1 ilustra a tela de gerenciamento das informações registradas pelo Sentinela: professores, alunos, turmas, links e situações das provas e registro de alertas (atividades suspeitas).

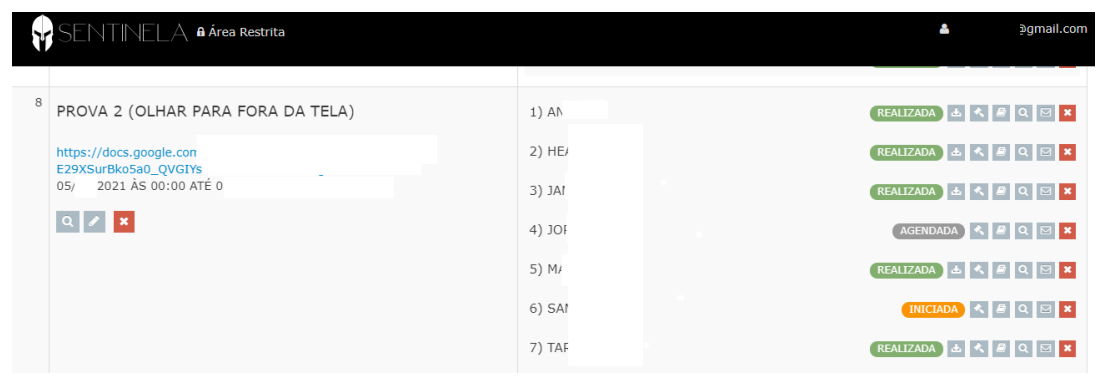

Figura 1. Tela de gerenciamento do sistema Sentinela

Outra funcionalidade do Sentinela é o registro e gerenciamento de informações relacionadas à interação do estudante: início, término e duração da prova; número de tentativas de saída/retorno à janela da prova; e o tempo decorrido entre a saída e o retorno à janela de prova.

Para realizar a prova, cada estudante acessa um link individual enviado por email que dá acesso ao ambiente digital. Ao acessar pela primeira vez, o sistema exibe os dados de identificação da prova e do estudante, registra o IP e a data/hora do acesso, e solicita o aceite ao termo de livre consentimento para participar desta pesquisa. A Figura 2 apresenta essa etapa. Logo em seguida surge uma tela na qual há um vídeo mostrando o rosto do estudante utilizando a webcam do computador e possibilitando que ele se posicione corretamente. Logo abaixo há um botão para iniciar a prova. Ao clicar para iniciar, é exibido o formulário Google Forms relacionado à atividade. Ao finalizar a avaliação, o estudante deve clicar em "Finalizar"para encerrar seu acesso à avaliação. 


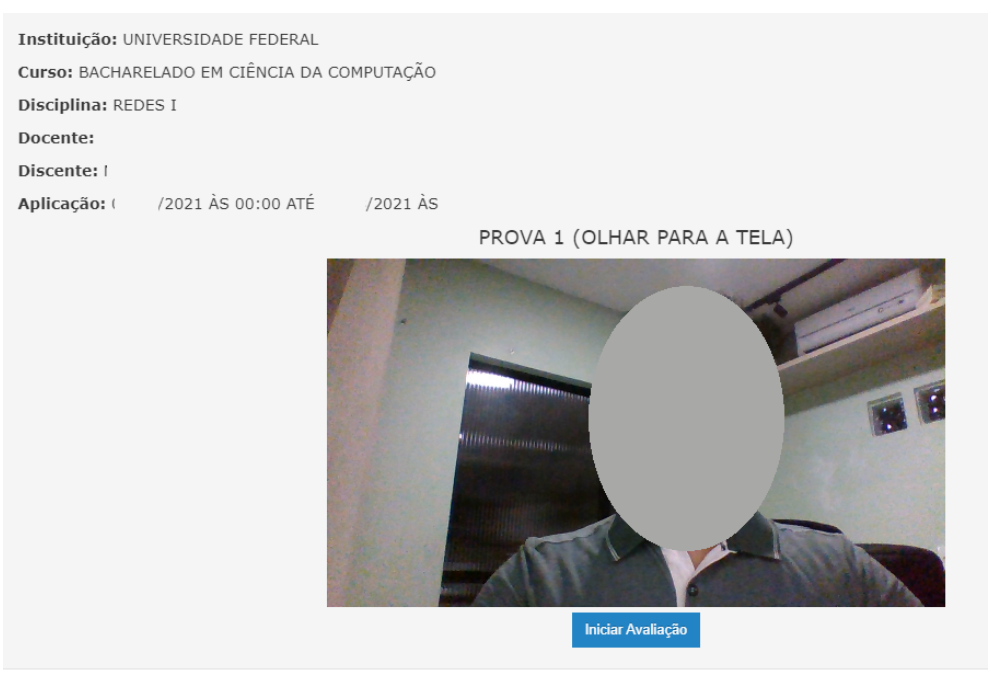

Figura 2. Tela de captura de imagens do estudante

\subsection{Redes Neurais Convolucionais}

A Rede Neural Convolucional (Convolutional Neural Network - CNN) é um tipo de aprendizado de máquina profundo com o propósito de detecção e classificação de objetos a partir de determinadas imagens [Braga et al. 2021]. Nesse aspecto, CNN é bastante utilizada para solucionar atividades associadas às imagens. Nesse trabalho foi utilizada a CNN LeNet-5 [Zhang et al. 2015], uma arquitetura composta por sete camadas. Com essa arquitetura foi treinado um modelo que serviu para mensurar a posição do olhar dos estudantes.

\section{Avaliação do Modelo}

A validação computacional do modelo proposto centrou-se na aplicação da ferramenta desenvolvida em um ambiente de ensino digital e online com os alunos da turma de um curso de pós-graduação em Computação. A seguir, na Figura 3, são apresentadas as etapas e técnicas computacionais expostas no modelo proposto, onde ilustra o método utilizado. Além disso, são apresentados os resultados obtidos com os estudantes.

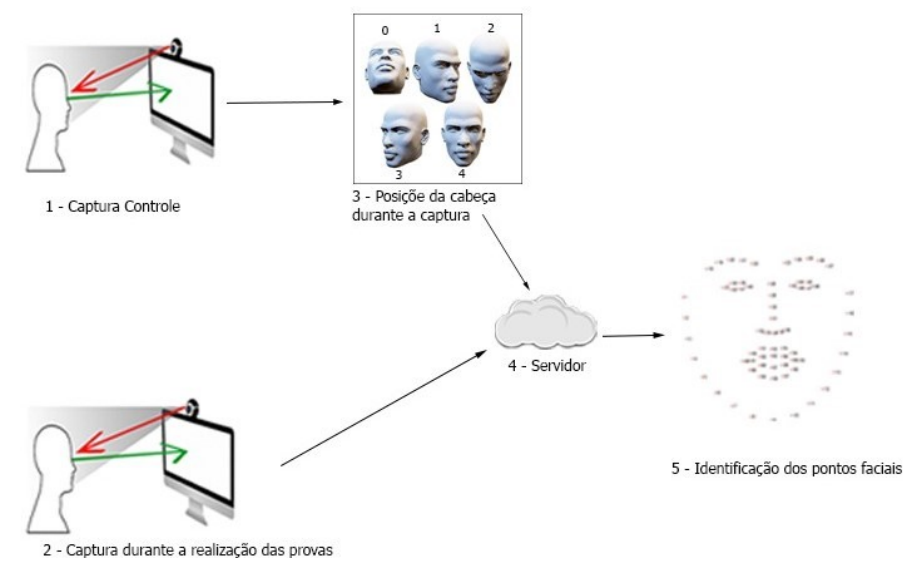

Figura 3. Método de captura do modelo proposto 


\subsection{Participantes}

Para avaliar o modelo, os participantes dos experimentos foram divididos em dois grupos: i) Grupo de Controle (GC) e o Grupo de Teste (GT). O Grupo de Controle (GC) foi formado por cinco participantes composto por membros da equipe da pesquisa. Esses membros são estudantes de pós-graduação em Ciências da Computação - Mestrado e Doutorado. Já o Grupo de Teste foi formado por 13 estudantes da disciplina de redes de computadores do curso de pós-graduação em Ciência da Computação.

Como apresentado, os estudantes são da área de Computação e assim têm o perfil direcionado à Tecnologia da Informação e não tiveram dificuldades em acessar o link da prova online e realizar o experimento. Esse procedimento foi simplificado na Figura 3. Vale frisar que ao acessar pela primeira vez o ambiente, o estudante foi direcionado a um termo de livre consentimento para participar desta pesquisa e em seguida pôde aceitar ou não tais termos.

\subsection{Captura das Imagens}

A captura das imagens de GC e GT foi realizada em dois processos diferentes. O processo de obtenção das imagens do GC foi realizado através da ferramenta Sentinela onde o participante foi instruído a olhar para 5 direções diferentes por 25 segundos enquanto a câmera registrava imagens do usuário olhando na posição solicitada. Com o GT, as imagens foram obtidas num processo de uso livre onde o participante em frente ao computador fez o uso da maneira habitual do dispositivo. Todos os participantes realizaram os procedimentos em computadores do tipo notebook com câmera integrada e ligada. A Figura 3 ilustra os processos de captura das imagens GC e GT.

\subsection{Rastreio do uso do navegador}

O rastreio do uso do navegador, durante a aplicação da avaliação, registra as seguintes informações: IP do dispositivo, plataforma (mobile, desktop), versões do navegador e do sistema operacional entre outras. Além disso, é feito o registro do tempo de prova, alertas de mudança de aba ou janela e tempo em que ficou fora da aba da prova. Nessa perspectiva, é possível comparar essas informações com situações anormais (divergentes) registradas na captura do movimento dos olhos.

\subsection{Classificação das imagens com $\mathrm{CNN}$}

Logo em seguida é feito o processo de treinamento utilizando CNN. Nesse cenário, foram obtidas 2.407 (duas mil, quatrocentas e sete) imagens. No referido treinamento utilizouse a base de dados obtida na etapa 1 (captura controle) apresentada na Figura 3 . A CNN utilizada baseou-se na LeNet5 apresentada por [Zhang et al. 2015]. Já a base de dados com as imagens capturadas durante a realização dos exames (etapa 2) foi usadas como base teste da CNN. Para o procedimento de avaliação do modelo, os pesquisadores rotularam 1000 (mil) imagens de cada participante em cinco classes. As classes rotuladas são classificadas em topo, à direita, rodapé, à esquerda e frontal à tela do computador.

O treinamento foi realizado em 1000 (mil) épocas e usou uma fatia de 80\%, 1926 (mil, novecentas e vinte e seis), imagens para treinamento, e 20\%, 481 (quatrocentas e oitenta e uma), imagens para validação. As imagens foram processadas para a identificação e recorte apenas das faces, redimensionando-as para 244x244 pixels. Obteve-se uma taxa 
de $97 \%$ de acurácia na validação da base de treinamento. Esse valor é significativo se comparado aos trabalhos citados na Seção2.

\section{Ameaças à validade do trabalho}

A escolha do modelo utilizado pode ser apontada como possível ameaça à validade do trabalho, pois já estão disponíveis outros modelos mais robustos. Entretanto, o modelo escolhido apresenta reduzido consumo de recursos computacionais em relação a outros modelos mais recentes.

\section{Resultados}

Nesta seção, serão apresentados os resultados oriundos dos experimentos com os participantes da pesquisa nas duas etapas. Nesse cenário, alguns resultados serão obtidos comparando os registros da captura da tela no momento da aplicação da prova com as imagens capturadas.

O primeiro resultado relevante é ilustrado na Figura 4. O gráfico apresenta a posição do olhar dos participantes em relação aos pontos da tela. Para isso, foram extraídas 1000 imagens dos rostos dos participantes de forma estocástica e rotulados os lados possíveis para os olhares dos estudantes para fora e dentro da tela do computador.

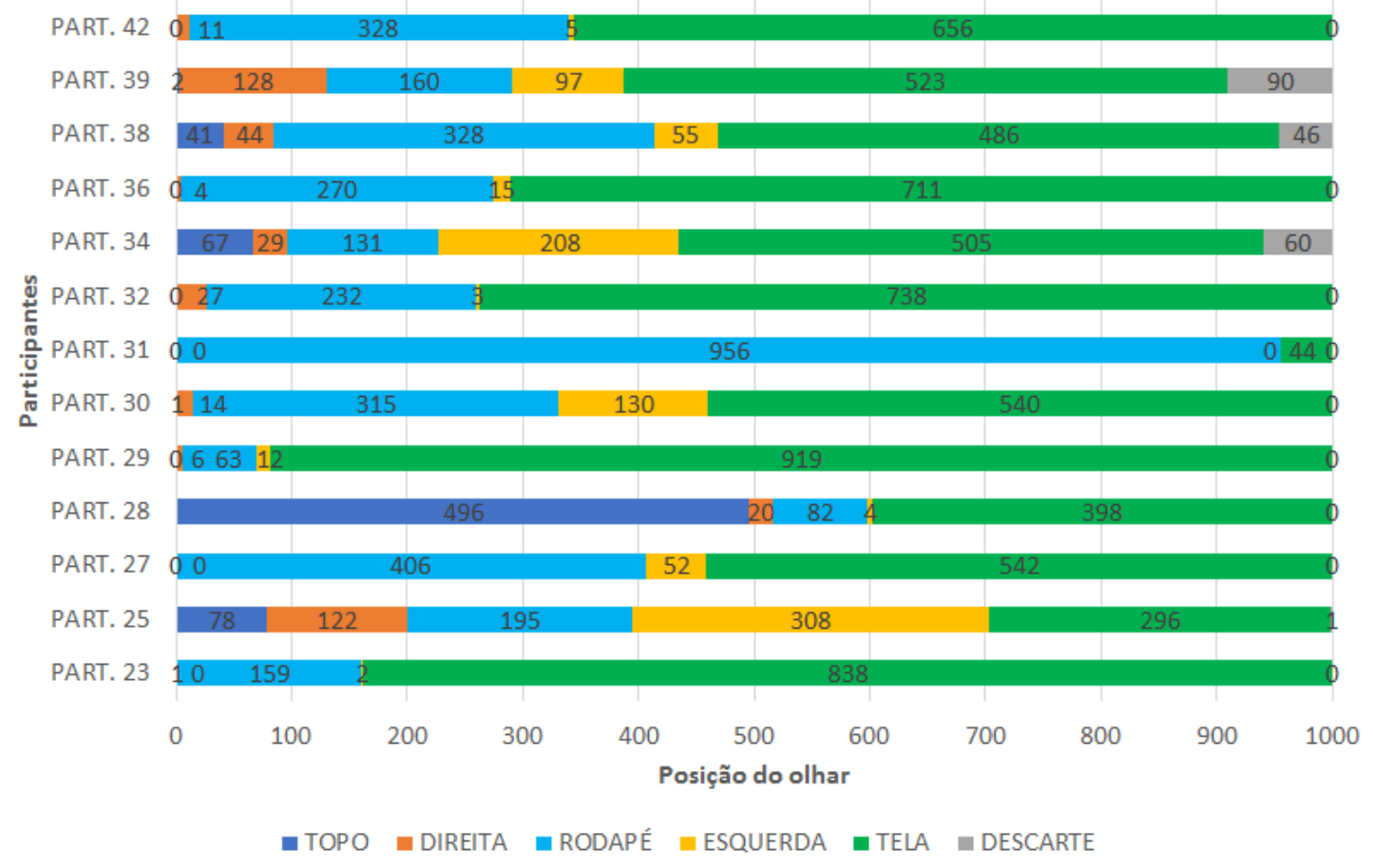

Figura 4. Gráfico com informações da posição do olhar

Avaliando ainda a Figura 4, infere-se que os estudantes passaram mais tempo olhando a tela. Além disso, foram descartadas imagens que não continham imagens dos estudantes ou imagens desfocadas.

Outro resultado sintomático é identificado quando se relaciona os dados de tempo de realização do exame, quantidade de alertas e o tempo que os alertas levaram. Essa relação é apresentada na Figura 5 . 


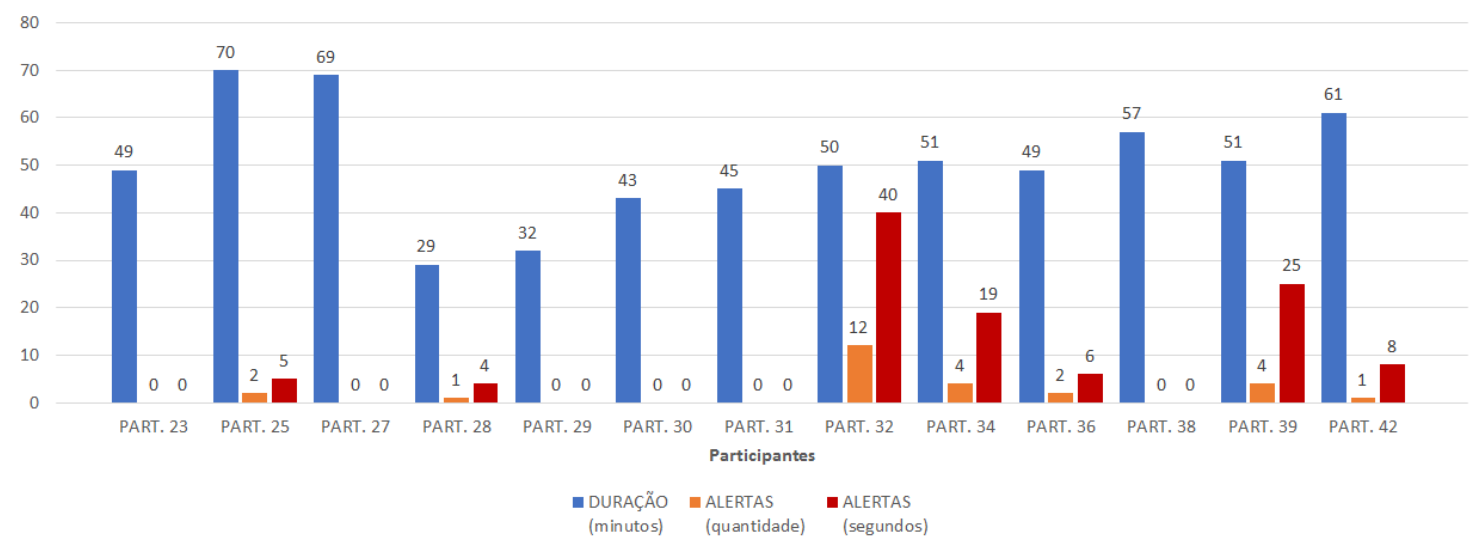

Figura 5. Gráfico confrontando duração de alertas x quantidade de alertas

Como visualizado na Figura 5, confrontou-se os dados de tempo de realização do exame, da quantidade de alertas e os tempos decorridos dos alertas. Um caso particular, observa-se no PART.32 com os valores: 50 minutos de realização do exame, 12 eventos de alerta e 40 segundos de duração destes alertas. Isso sugere um comportamento indevido durante a realização do exame. Essa relação pode indicar, por exemplo, que o participante pode ter entrado e saído várias vezes da plataforma.

Outra informação relevante explorada pela pesquisa é a duração da prova em relação à duração de alertas devido a troca de janela ativa pelo usuário. A Figura 6 apresenta o resultado encontrado para os 13 participantes. Destaca-se, de modo peculiar, o participante 32 que passou 40 segundos fora da tela da prova (em estado de alerta). Infere-se, nesse caso, possíveis tentativas de acessar conteúdo externo à avaliação.

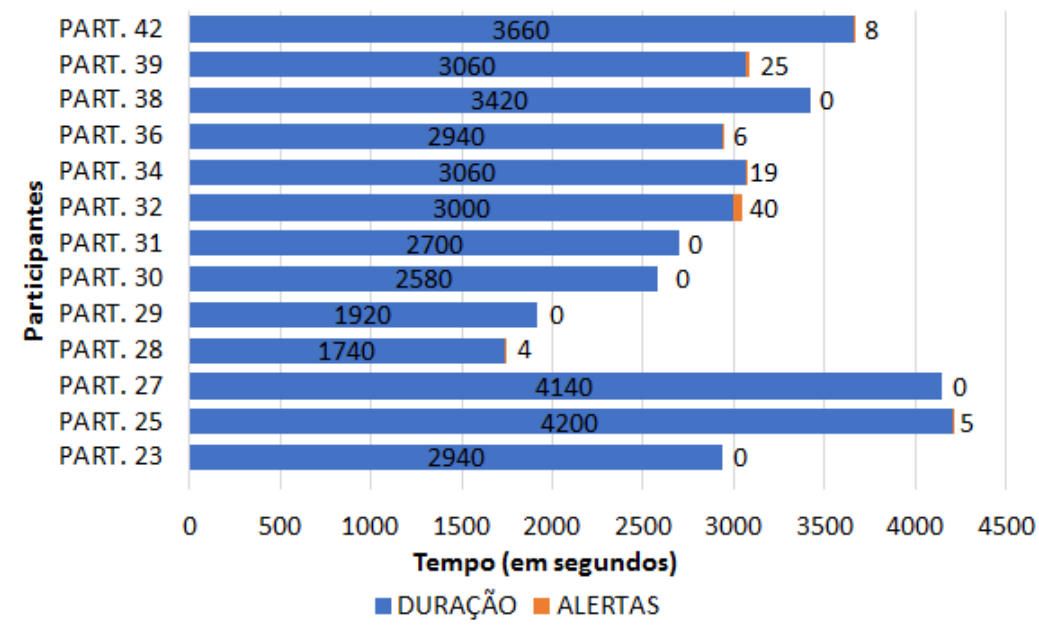

Figura 6. Duração da Prova

Quanto a acurácia (ou exatidão), a Figura 7 apresenta o resultado da classificação das imagens de cada estudante utilizando a CNN treinada. Nesse contexto, é possível notar que a maior acurácia obtida foi do estudante 29 com mais de $88 \%$. Observa-se estudantes com taxa de acurácia baixa, a exemplo o estudante 31 com menos de $20 \%$. Estes números sugerem a possibilidade de utilizar uma outra CNN para classificar as imagens 
obtidas dos estudantes. Não obstante, é necessário um trabalho minucioso na obtenção das imagens como iluminação e posicionamento da câmera, além de um processamento diligente das imagens dos estudantes.

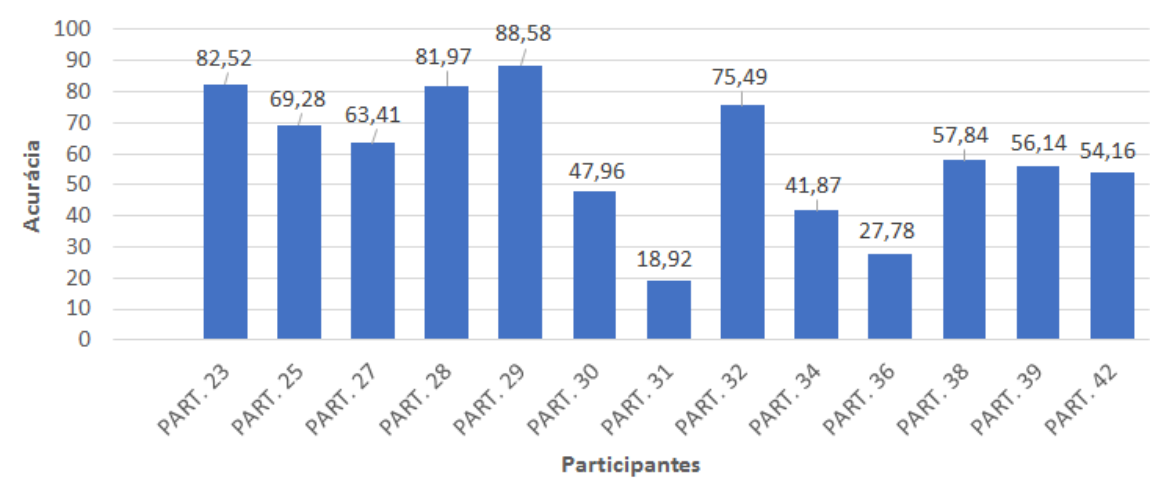

Figura 7. Acurácia por participante

Mediante o exposto, vale ressaltar que é possível confrontar outros dados expostos nesta pesquisa. Nesse sentido, pode-se obter diversos outros resultados relevantes sobre o comportamento dos alunos na aplicação da prova online.

\section{Considerações Finais}

Neste trabalho foi apresentada uma estratégia que auxilia o professor no monitoramento de exames online no contexto de ensino na modalidade remota. Nessa perspectiva, foi realizada a captura das imagens dos estudantes e informações sobre o registro de acesso às janelas de prova, com a possibilidade de registrar informações de acesso a outras janelas. Os resultados foram relevantes pois possibilitam ao professor cotejar os dados capturados na prova com os resultados obtidos nas avaliações das imagens capturadas dos estudantes.

Para trabalhos futuros, almeja-se padronizar o uso da webcam, os posicionamentos dos usuários e tela. Além disso, outras informações serão coletadas, tais como objetos na tela, áudio do usuário, cliques de mouse, entre outros, assim como a verificação de outras estruturas de CNN disponíveis.

\section{Referências}

[Asep and Bandung 2019] Asep, H. S. and Bandung, Y. (2019). A design of continuous user verification for online exam proctoring on m-learning. In 2019 International Conference on Electrical Engineering and Informatics (ICEEI), pages 284-289. IEEE.

[Braga et al. 2021] Braga, M., e Silva, W. S., Junior, O. O., and Torres, R. (2021). Análise de sentimento com rede neural convolucional: uma investigação do fator motivacional da metodologia de aprendizagem criativa. In Anais do XLVIII Seminário Integrado de Software e Hardware, pages 191-200, Porto Alegre, RS, Brasil. SBC.

[Hu et al. 2018] Hu, S., Jia, X., and Fu, Y. (2018). Research on abnormal behavior detection of online examination based on image information. In 2018 10th International Conference on Intelligent Human-Machine Systems and Cybernetics (IHMSC), volume 2, pages 88-91. IEEE. 
[Indi et al. 2021] Indi, C. S., Pritham, K., Acharya, V., and Prakasha, K. (2021). Detection of malpractice in e-exams by head pose and gaze estimation. International Journal of Emerging Technologies in Learning, 16(8).

[Li et al. 2021] Li, H., Xu, M., Wang, Y., Wei, H., and Qu, H. (2021). A visual analytics approach to facilitate the proctoring of online exams. In Proceedings of the $2021 \mathrm{CHI}$ Conference on Human Factors in Computing Systems, pages 1-17.

[Muzaffar et al. 2021] Muzaffar, A. W., Tahir, M., Anwar, M. W., Chaudry, Q., Mir, S. R., and Rasheed, Y. (2021). A systematic review of online exams solutions in e-learning: Techniques, tools, and global adoption. IEEE Access, 9:32689-32712.

[Prathish et al. 2016] Prathish, S., Bijlani, K., et al. (2016). An intelligent system for online exam monitoring. In 2016 International Conference on Information Science (ICIS), pages 138-143. IEEE.

[Vieira and Silva 2020] Vieira, M. and Silva, C. (2020). A educação no contexto da pandemia de covid-19: uma revisão sistemática de literatura. Revista Brasileira de Informática na Educação, 28(0):1013-1031.

[Zhang et al. 2015] Zhang, X., Sugano, Y., Fritz, M., and Bulling, A. (2015). Appearancebased gaze estimation in the wild. In Proceedings of the IEEE conference on computer vision and pattern recognition, pages 4511-4520. 